\title{
Detection and Classification of Voltage Disturbances in Electrical Power Systems using a Modified Euclidean ARTMAP Neural Network with Continuous Training
}

Ana Claudia Barros, Mauro S. Tonelli-Neto, José Guilherme Magalini Santos Decanini \& Carlos Roberto Minussi

To cite this article: Ana Claudia Barros, Mauro S. Tonelli-Neto, José Guilherme Magalini Santos Decanini \& Carlos Roberto Minussi (2015) Detection and Classification of Voltage Disturbances in Electrical Power Systems using a Modified Euclidean ARTMAP Neural Network with Continuous Training, Electric Power Components and Systems, 43:19, 2178-2188, DOI: 10.1080/15325008.2015.1073814

To link to this article: https://doi.org/10.1080/15325008.2015.1073814

\section{曲 Published online: 14 Sep 2015.}

Submit your article to this journal

Llll Article views: 130

View Crossmark data \lceil

Citing articles: 2 View citing articles $\widetilde{ }$ 


\title{
Detection and Classification of Voltage Disturbances in Electrical Power Systems Using a Modified Euclidean ARTMAP Neural Network with Continuous Training
}

\author{
Ana Claudia Barros, ${ }^{1}$ Mauro S. Tonelli-Neto, ${ }^{2}$ José Guilherme Magalini Santos \\ Decanini, ${ }^{3}$ and Carlos Roberto Minussi ${ }^{1}$ \\ ${ }^{1}$ Electrical Engineering Department, Câmpus of Ilha Solteira, Univ Estadual Paulista, Ilha Solteira, SP, Brazil \\ ${ }^{2}$ Instituto Federal de Educação, Ciência e Tecnologia de São Paulo, Câmpus Votuporanga, Votuporanga, SP, Brazil \\ ${ }^{3}$ Instituto Federal de Educação, Ciência e Tecnologia de São Paulo, Câmpus Presidente Epitácio, Presidente Epitácio, SP, Brazil
}

\section{CONTENTS}

\section{Introduction}

2. Structure of the Proposed Methodology

3. WT

4. MRA

5. Disturbance Detection Module

6. Feature Extraction Module

7. Processing and Codification Module

8. Disturbance Classification Module Using MECT-ARTMAP

9. Test System

10. Results and Discussions

11. Conclusion

Funding

References
Keywords: wavelet transform, Euclidean ARTMAP neural network, continuous training, power quality disturbance, power distribution system Received 30 May 2014; accepted 22 June 2015

Address correspondence to Dr. Carlos Minussi, Electrical Engineering Department, Câmpus of Ilha Solteira, UNESP, Univ Estadual Paulista, Av. Brasil 56, 15385-000 Ilha, Solteira, SP, Brazil. E-mail:

minussi@dee.feis.unesp.br
Abstract - This article presents a method to detect and classify voltage disturbances in electric power distribution systems using a modified Euclidean ARTMAP neural network with continuous training. This decision-making tool accelerates the procedures to restore the normal operation conditions providing security, reliability, and profits to utilities. Furthermore, it allows the diagnosis system to adapt to changes from the constant evolution of the electric system. The voltage signals features or signatures are extracted using discrete wavelet transform, multiresolution analysis, and the energy concept. Results show that the proposed methodology is robust and efficient, providing a fast diagnosis process. The data set used to validate the proposal is obtained by simulations in a real distribution system using ATP software.

\section{INTRODUCTION}

Users have recently been demanding a best energy supply with quality and reliability, reducing voltage abnormalities and minimizing interruptions caused by disturbances $[1,2]$. Among the most common disturbances, emphasis is on voltage sag; caused by, e.g., short circuit, voltage swell; caused by, e.g., capacitor bank switching, and harmonics; caused by, e.g., the presence of non-linear equipment connected to the electric network.

The occurrence of these disturbances degrade the power quality, leading to faulty operation of some equipment connected to the network, conductor superheating, and malfunction of the protection system. Therefore, distribution companies invest in developing new techniques that are able to detect and classify such disturbances quickly, with security and in an efficient manner, contributing to the automation of the distribution systems [3, 4]. Normally, detection of the disturbances is done through the visual inspection of voltage disturbances by the operators, who require some previous experience 
in analysis and decision making. Because of the dependence on human operators, the diagnosis is susceptible to errors. Thus, the automation of this practice is very important, where techniques based on artificial intelligence assist the operator to classify abnormalities.

The specialized literature contains several proposals aiming to identify voltage disturbances automatically $[5,6,7,8]$.

In [9], a hybrid technique to characterize the power quality disturbances is presented. The hybrid technique uses the Kalman filter with discrete wavelet transform (DWT) to extract the waveform characteristics. Then, based on fuzzy rules, the fuzzy system provides as output the type of the disturbance.

A new approach to classify the power quality disturbances employing the Markov model and wavelet transform (WT) was proposed in [10]. By applying WT, it is possible to calculate the energy distribution coefficients considering each decomposition level of the analyzed disturbances. Therefore, the extracted indexes initialize the training matrix of the Markov model maximizing the precision of the classification. Furthermore, the Dempster-Shafer algorithm [10] is used to obtain qualitative information about the result.

In [11], a classification system based on S-transform and a probabilistic neural network was proposed. The power quality disturbances are analyzed by S-transform; then the characteristics of the time-frequency domain are extracted from the S-matrix to compose the input vector of the neural system, which provides the diagnosis.

In [12], a base rule was employed to classify the power quality disturbance. The disturbances are characterized by multilayer S-transform, which acts as a singularity extraction tool. The pattern classification algorithm that identifies the disturbances uses linear and parabolic rules, where the decision intervals are defined by heuristics.

In [13], a methodology was proposed to classify power quality disturbances by an intelligent recognition system. The characteristics representing the disturbances are extracted applying WT on the three-phase voltage signals. Also, a support vector machine classifies the disturbance present on the power system.

In [14], a decision-making system was presented for automated classification of power quality disturbances, where the most common types of disturbances, including flickers, harmonics, impulses, notches, outages, sags, swells, and switching transients, were studied. The signal-processing techniques utilized to extract the waveforms features are Fourier and Stransform, and a method based on the binary feature matrix was designed to classify the disturbance type.

Considering the concept of the smart grid, where the distribution systems must be intelligent and with learning capac- ity, the development of voltage disturbance diagnosis methods must present the above-described characteristics. Selfadaptation is a fundamental characteristic that allows the system to adapt to the changes coming from the expansion and evolution of the electrical system, e.g., new kinds of disturbances, failures, and without the interference of human operators. Considering that several techniques based on artificial intelligence are available, some characteristics in choosing one or other must be observed. For example, none allows activating an automatic learning system without the interference of human operators. Therefore, the ART family neural networks that present characteristics of plasticity and stability can be used to incorporate new information different from those previously acquired, i.e., knowledge.

This study presents an auto-adaptive methodology for voltage disturbance diagnosis in electrical distribution feeders. It is simple, robust, and flexible, using the modified Euclidean adaptive resonance theory mapping neural network, to classify and learn different disturbances [15]. The neural network is based on a new conception where the performance is improved as time goes by; i.e., as new knowledge is presented to the network, it is not necessary to reinitialize the training process. The reinforcement of this knowledge is on-line, automatic and refines the previously acquired knowledge once the network presents parameters that identify the presence of new knowledge. This continuous training module does not require additional computational effort when compared to other neural network configurations, e.g., the backpropagation algorithm.

The continuous training uses data of the acquisition system (supervisory control and data acquisition [SCADA]), data obtained from simulations containing topology changes on the electrical system, and other kinds of voltage disturbance, including other abnormalities, e.g., low- and high-impedance short circuits.

The extraction of the voltage waveform characteristics is realized through the employment of DWT, multi-resolution analysis (MRA), and the energy concept. These characteristics correspond to the input vector of the MECT-ARTMAP neural network. As output, the neural network provides a binary codification previously defined to represent the type of disturbance present on the feeder.

This article is organized as follows. Section 2 shows the structure of the method used. Sections 3 and 4 present the DWT and the MRA, respectively. The voltage disturbance detection module is described in Section 5. Section 6 emphasizes the procedures to extract the voltage disturbance characteristics. Section 7 shows the methodology to process and codify the voltage disturbances. Section 8 presents the classification module. Section 9 highlights the database configuration. The 


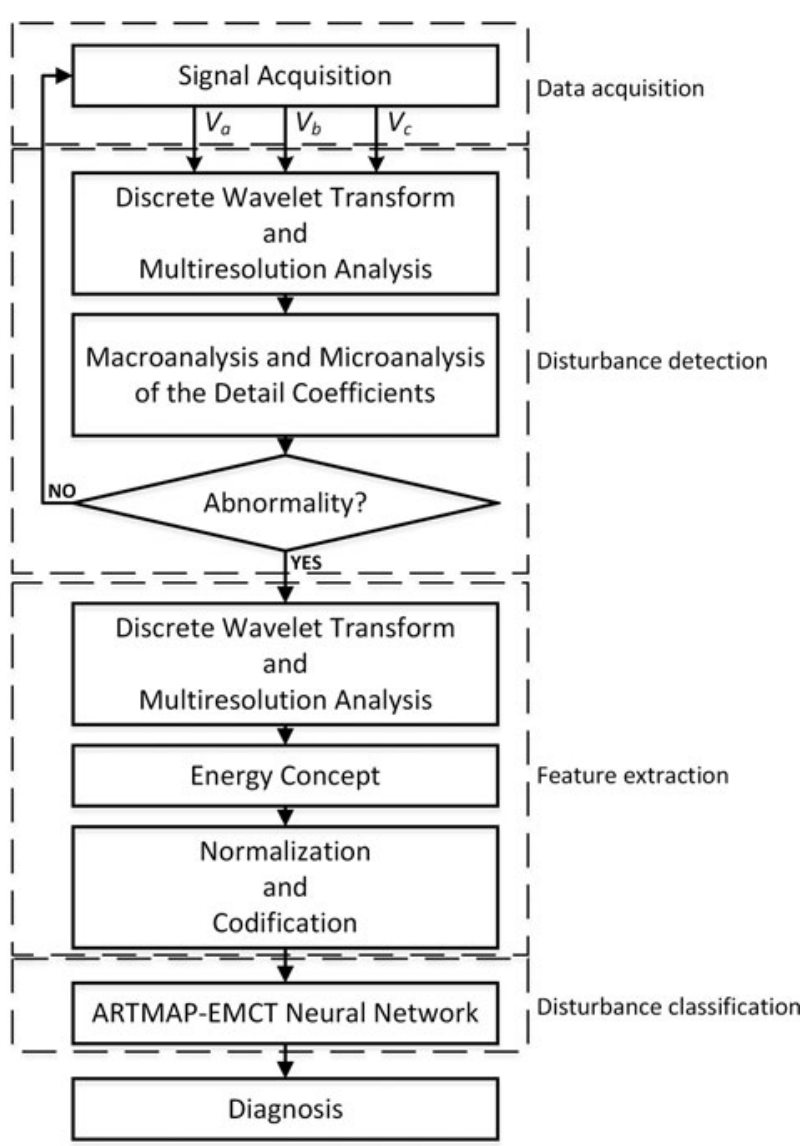

FIGURE 1. Structure of the voltage abnormality diagnosis system.

results and discussions are presented in Section 10 and the conclusion in Section 11.

\section{STRUCTURE OF THE PROPOSED METHODOLOGY}

Figure 1 presents the modules of the developed system to detect and classify voltage disturbances. This system can be modified to attend the necessities of distribution companies according to the electrical system constant evolution.

\section{WT}

In most applications, where it is necessary to extract characteristics, the methods employed are based on Fourier transform. Even being a powerful tool for disturbance diagnosis, Fourier transform is inadequate to analyze non-stationary signals. Thus, WT is a concept that surpasses the limitations of techniques based on Fourier transform once it provides the temporal evolution of frequency transients [16].
WT is a linear transform that uses oscillation functions with different frequencies as windowing functions. This base function, called the mother wavelet, must have finite energy and medium value equal to zero. The mother wavelet is defined according to Eq. (1) [17]:

$$
\psi_{r, s}(t)=\frac{1}{\sqrt{|r|}} \psi\left(\frac{t-s}{r}\right),
$$

where $s$ is a translation parameter and $r$ the scale parameter.

To analyze oscillographs obtained by acquisition data equipment, it is necessary to discretize the wavelet function. Thus, DWT of a sample signal $f(k)$ is defined as follows [17]:

$$
D W T_{f}=\left\langle f, \psi_{m, n}\right\rangle=\sum_{k} f[k] \psi_{m . n}^{*}[k],
$$

where

$$
\psi_{m, n}^{*}[k]=\frac{1}{\sqrt{r_{0}^{m}}} \psi^{*}\left[\frac{k-n s_{0} r_{0}^{m}}{r_{0}^{m}}\right]
$$

\section{MRA}

MRA consists of calculating coefficients $c a_{m}$ and $c d_{m}$ determined by Eqs. (3) and (4), respectively. To calculate coefficients $c a_{m+1}$ and $c d_{m+1}$, it is necessary to perform the discrete convolution of the signal $c a_{m}$ with a low-pass filter ( $h$; i.e., discrete scale function) and a high-pass filter ( $g$; i.e., discrete wavelet function). At the output of each one of the filters, the decomposed signal is subsampled by a factor of 2 [18]:

$$
\begin{aligned}
& c a_{m+1}[p]=\sum_{n} h[n-2 p] c a_{m}[n], \\
& c d_{m+1}[p]=\sum_{n} g[n-2 p] c a_{m}[n] .
\end{aligned}
$$

The coefficients obtained at the output of the low-pass filter $c a_{m+1}$ are characterized as being the high-scale and low-frequency components of the signal, called approximation coefficients. The coefficients obtained at the output of the high-pass filter $c d_{m+1}$ are the components of low-scale and high-frequency components, called detail coefficients.

\section{DISTURBANCE DETECTION MODULE}

The voltage disturbance detection module is designed considering the constant evolution of technologies employed at the substations and the natural dynamism of the electric power systems, such as loading, disturbance insertion angle, location, and noise presence in oscillographs, and is aimed to obtain a robust, flexible, and efficient methodology. 
The disturbance detection is done by analyzing the voltage oscillographs acquired at the principal substation output. In this step, the signal windowing is defined to be two cycles and the step under analysis to be one cycle. Thus, MRA is applied to decompose the signal of the phase in two resolution levels. Subsequently, calculations of the arithmetic average of the absolute value of the detail coefficients (previously defined in Eq. (4)) of each one of the decomposition level of the oscillograph under analysis are made [16]:

$$
\overline{d_{i j}}=\frac{\sum_{k=1}^{N_{j}}\left|c d_{i j}^{k}\right|}{N_{j}},
$$

where

$i$ is the oscillograph under analysis, i.e., $V a, V b$ and $V c$;

$j$ is the decomposition level, i.e., 1 and 2;

$N_{j}$ is the quantity of the detail coefficients of level $j$;

$k$ is the $k$ th detail coefficient of level $j$ referred to oscillograph $i$; and

$\bar{d}_{i j}$ is the arithmetic average of the detail coefficients of level $j$ referred to oscillograph $i$.

Further, the variation in relation to the arithmetic average obtained for each detail coefficient using Eq. (6) is calculated [16]:

$$
\operatorname{var}_{i j}^{k}=|| c d_{i j}^{k}\left|-\overline{d_{i j}}\right|,
$$

where $\operatorname{var}_{i j}^{k}$ is the variation of the $k$ th detail coefficient in relation to the arithmetic average of these coefficients for level $j$ referred to oscillograph $i$.

At the same time, the standard deviation of the absolute value of the detail coefficients under evaluation is obtained [16]:

$$
\sigma_{i j}=\sqrt{\frac{\sum_{k=1}^{N_{j}}\left(\left|c d_{i j}^{k}\right|-\overline{d_{i j}}\right)^{2}}{N_{j}-1}},
$$

where $\sigma_{i j}$ is the standard deviation of the detail coefficients of level $j$ referred to oscillograph $i$.

The voltage disturbance detection process is based on the two sets of rules presented in Eqs. (8) and (9), where each rule is divided into two parts: macro-analysis and micro-analysis. The general behavior of the signal is known by the macroanalysis, i.e., comparing the maximal variation of the detail coefficients with a percentage of the standard deviation, which provides major immunity to noise presence in oscillographs under analysis and minimizes the effect of changes in the power distribution system. The micro-analysis allows a point-to-point analysis of the major detail coefficient with a predefined limit (set by the operator) [16]:

$$
\text { if } \max \left[\boldsymbol{v a r}_{i j}\right]>1.25 \sigma_{i j} \max \left[\boldsymbol{c d} \boldsymbol{d}_{i j}\right]>\mu_{j} \text {, then } \tau_{i j}=1
$$

shows the existence of abnormalities on the system

$$
\text { if } \max \left[\boldsymbol{v a r}_{i j}\right] \leq 1.25 \sigma_{i j} \text { or } \max \left[\boldsymbol{c d} \boldsymbol{d}_{i j}\right] \leq \mu_{j} \text {, then } \tau_{i j}=0
$$

shows the normal operation of the system, where $\mu_{j}$ is the parameter to be specified based on the protection philosophy of the system and the operator experience, and $\tau_{i j}$ is the index of the operation state of the system.

The rate 1.25 is defined after the analyzing the values from Eqs. (6) and (7) and also considering the signals representing the normal operation from the voltage disturbances. Thus, the detection module presented in Figure 1 assures the presence of abnormalities if the rule of Eq. (9) is satisfied, and the normal operation if the rule of Eq. (8) is satisfied.

\section{FEATURE EXTRACTION MODULE}

Once any abnormality on the system is detected, the classification step is activated. The extraction of the disturbance characteristic indices is realized by DWT, MRA, and the energy concept. These behavioral indices compose the input vector of the MECT-ARTMAP, which classifies the disturbance.

\subsection{Energy}

The energy concept corresponds to a data fusion tool that can be used to aggregate information extracted from a signal through signal processing techniques. In particular, this concept is used often to aggregate the coefficients extracted using DWT. If the diagnosis procedure considers these features as input, the process can become inefficient once high computational effort is required. Therefore, the application of the energy concept reduces the dimension of the input vector; i.e., the coefficients are aggregated in scalars, leading the procedure to be faster and reliable [19].

The energy can be calculated using the following equation:

$$
E=\sum_{n_{p}=1}^{Z}\left|x\left[n_{p}\right]\right|^{2},
$$

where $x\left[n_{p}\right]$ is the $n_{p}$ th sample of signal $x, Z$ is the quantity of points of the analyzed signal, and $E$ is the energy of the signal.

\subsection{Characteristic Extraction}

The classification model selects two pre-disturbance and two disturbance cycles of the voltage oscillographs. These cycles are decomposed by the MRA into four resolution levels. It is emphasized that DWT is applied again once the cycles are analyzed at this juncture. It is to be noted that these two pre- 
disturbance cycles and two cycles with disturbance are different from those used on the detection process.

The characteristics of the system's operation state are based on the detail coefficients of the third and fourth level, and the approximation coefficients of the fourth level [20]. Then the energy of the detail and approximation coefficients of the pre-disturbance cycles, considering each one of the phases, is calculated according to Eqs. (11) and (12):

$$
\begin{aligned}
& E c a_{j}^{f, p d}=\sum_{n=1}^{N j}\left|c a_{j}^{f, p d}[n]\right|^{2}, \\
& E c d_{j}^{f, p d}=\sum_{n=1}^{N j}\left|c d_{j}^{f, p d}[n]\right|^{2},
\end{aligned}
$$

where

$p d$ is the pre-disturbance;

$j$ is the decomposition level, i.e., third and fourth detail level, and fourth approximation level;

$f$ is the phase of the analyzed oscillograph, i.e., $V a, V b$, and $V c$;

$N_{j}$ is the coefficients' quantity of level j;

$c a^{p d}[n]$ is the value of the $n$th approximation coefficient of the pre-disturbance cycles; and

$c d^{p d}[n]$ is the value of the $n$th detail coefficient of the predisturbance cycles.

The same procedure is applied for the coefficients of the disturbance cycles:

$$
\begin{aligned}
& E c a_{j}^{f}=\sum_{n=1}^{N_{j}}\left|c a_{j}^{f}[n]\right|^{2}, \\
& E c d_{j}^{f}=\sum_{n=1}^{N_{j}}\left|c d_{j}^{f}[n]\right|^{2},
\end{aligned}
$$

where:

$f$ is the phase of the analyzed oscillograph, i.e., $V a, V b$, and $V c$;

$j$ is the decomposition level, i.e., third and fourth detail level, and fourth approximation level;

$N j$ is the coefficients' quantity of level $\mathrm{j}$;

$c a[n]$ is the value of the $n$th approximation coefficient of the cycles with disturbance; and

$c d[n]$ is the value of the $n$th detail coefficient of the cycles with disturbance.

Thus, the characteristic vectors for the pre and with disturbance cycles are defined.

$$
\boldsymbol{A}_{\text {pre-disturbance }}=\left[E_{c d 3}^{V a, p d} E_{c d 4}^{V a, p d} E_{c a 4}^{V a, p d} E_{c d 3}^{V b, p d} E_{c d 4}^{V b, p d}\right.
$$

$$
\begin{array}{r}
\left.E_{c a 4}^{V b, p d} E_{c d 3}^{V c, p d} E_{c d 4}^{V c, p d} E_{c a 4}^{V c, p d}\right], \\
A_{\text {disturbance }}=\left[E_{c d 3}^{V a} E_{c d 4}^{V a} E_{c a 4}^{V a} E_{c d 3}^{V b} E_{c d 4}^{V b} E_{c a 4}^{V b} E_{c d 3}^{V c} E_{c d 4}^{V c} E_{c a 4}^{V c}\right],
\end{array}
$$

Subsequently, the pre-disturbance characteristic vector is subtracted from the characteristic vector under disturbance. This is necessary to obtain a complete analysis of the type of disturbance, incorporating into the methodology system's reference operation, i.e., normal operation [20]:

$$
\boldsymbol{\Gamma}=\boldsymbol{A}_{\text {disturbance }}-\boldsymbol{A}_{\text {pre-disturbance }} \text {. }
$$

After analyzing the type of disturbance, vector $\Gamma$ is normalized and codified to obtain more generalization of the project and maintaining the signal information (positive and negative) [20].

\section{PROCESSING AND CODIFICATION MODULE}

The input vector of the MECT-ARTMAP neural network (a) is defined by [20]

$$
a=[\Psi \Phi]
$$

where

$$
\boldsymbol{\Psi}=\left[\Psi_{1} \Psi_{2} \ldots \Psi_{9}\right]
$$

with

$$
\begin{gathered}
\Psi_{i}=\frac{\left|\Gamma_{i}\right|}{\Gamma_{\max }}, \\
\left|\Gamma_{\max }\right|=\max \left\{\left|\Gamma_{i}\right|\right\}, i=1, \ldots, 9 .
\end{gathered}
$$

The components of vector $\phi$ are defined according to

$$
\left\{\begin{array}{l}
\Psi_{i}>0 \rightarrow \Phi_{i}=1 \\
\Psi_{i} \leq 0 \rightarrow \Phi_{i}=0
\end{array}, i=1, \ldots, 9\right.
$$

where $\psi$ is the vector of the normalized characteristics, and $\phi$ is the signal of each normalized characteristic.

This process corresponds to the normalization step presented in Figure 1. This procedure must be executed for each vector to be analyzed to provide a correct distinction among the different disturbances analyzed by the modified Euclidean ARTMAP (ME-ARTMAP) neural network.

The output of the neural network, defined by vector $(\boldsymbol{b})$, provides the type of voltage disturbance present on the system. This output is codified as shown on Table 1. 


\begin{tabular}{|c|c|}
\hline Voltage disturbance & Output codification \\
\hline Swell & {$\left[\begin{array}{llll}0 & 0 & 0 & 1\end{array}\right]$} \\
\hline Sag & {$\left[\begin{array}{llll}0 & 0 & 1 & 0\end{array}\right]$} \\
\hline Interruption & {$\left[\begin{array}{llll}0 & 0 & 1 & 1\end{array}\right]$} \\
\hline Harmonics & {$\left[\begin{array}{llll}0 & 1 & 0 & 0\end{array}\right]$} \\
\hline Swell with harmonics & {$\left[\begin{array}{llll}0 & 1 & 0 & 1\end{array}\right]$} \\
\hline Sag with harmonics & {$\left[\begin{array}{llll}0 & 1 & 1 & 0\end{array}\right]$} \\
\hline Oscillatory transient & {$\left[\begin{array}{llll}0 & 1 & 1 & 1\end{array}\right]$} \\
\hline
\end{tabular}

TABLE 1. Output codification

\section{DISTURBANCE CLASSIFICATION MODULE USING MECT-ARTMAP}

The classification of the voltage disturbances is realized by the MECT-ARTMAP, which had been previously training in the off-line mode to improve the acquired knowledge in the on-line mode, i.e., during the diagnosis.

\subsection{ME-ARTMAP Neural Network}

The ME-ARTMAP is a self-organized system composed of two Euclidean adaptive resonance theory (ART) modules, $\mathrm{ART}_{\mathrm{a}}$ and $\mathrm{ART}_{\mathrm{b}}$, interconnected by an associate memory module called inter-ART. This neural network uses the Euclidean distance in the calculus, allowing the neural system responses to binary or analogic input patterns, pertaining to the set $\mathrm{R}^{+}$ $([0,+\infty])$.

The inter-ART module has a self-regulatory mechanism, called match-tracking, that uses the fuzzy operator AND $(\wedge)$ to verify if the matching with the input and output of the neural network happens or not, as well as that at ARTMAP-fuzzy [21]. If there is no matching, then the reset occurs. At the reset, a new category of the $\mathrm{ART}_{\mathrm{a}}$ module is chosen to be introduced at the resonance process. Furthermore, a decrease occurs at the parameter $\rho_{a}$. The inter-ART vigilance criterion is only activated when no category is created either in $\mathrm{ART}_{\mathrm{a}}$ or in $\mathrm{ART}_{\mathrm{b}}$. It is initialized with one active neuron, i.e., the first pattern presented to the network $\left(\boldsymbol{W}_{1, i}^{a}=\boldsymbol{a}_{1, i}\right.$ and $\left.\boldsymbol{W}_{1, j}^{b}=\boldsymbol{b}_{1, j}\right)$. The weights of the inter-ART module are defined as follows: $W_{1,1}^{a b}=1$ and $W_{1, n}^{a b}=0$, where $n=2,3, \ldots, N$. The other weights $\boldsymbol{W}^{a b}$ are set in 1 [22]. The input and output vectors are represented by $\boldsymbol{a}$ and $\boldsymbol{b}$, respectively, and defined according to Eqs. (20) and (21):

$$
\begin{aligned}
& \boldsymbol{a}=\left[a_{1}, a_{2}, \ldots, a_{M a}\right], \\
& \boldsymbol{b}=\left[b_{1}, b_{2}, \ldots, b_{M b}\right],
\end{aligned}
$$

where

$$
a_{i} \in[0,1], i=1,2, \ldots, M a,
$$

$$
b_{j} \in[0,1], j=1,2, \ldots, M b,
$$

where $M a$ is the dimension of vector $\boldsymbol{a}$ (input), $M b$ is the dimension of vector $\boldsymbol{b}$ (output), and $N$ is the quantity of input and output patterns.

The training depends basically on choosing the vigilance parameters $\left(\rho_{a}, \rho_{b}\right.$, and $\left.\rho_{a b}\right)$, the training rate $(\beta)$, and the increasing parameter $(\varepsilon)$.

The ME-ARTMAP neural network is a conception that provides solutions with quality, precision, and speed. One advantage of this neural network is that the complementary normalization and codification of the input patterns are not required, which consequently doubles the dimension of the input vector. Furthermore, as it uses a quadratic geometry, the formation of classes is more precise when compared to the conventional formulation of the fuzzy ARTMAP (rectangular geometry). Figure 2 presents the flowchart of the ME-ARTMAP neural network.

\subsection{MECT-ARTMAP Neural Network}

The continuous training to be incorporated at the ME-ARTMAP neural network is presented as follows. With the training in a continuous form, if new patterns are available, e.g., simulations or oscillographs provided by the data acquisition system, the training step does not need to be reinitialized, which is different when compared to other neural network approaches already known in the literature. The incorporation of continuous training, as in the ME-ARTMAP and other ART family neural networks, is possible because of the stability and plasticity characteristic, consisting of an improved neural system when compared to the multi-layer perceptron with the backpropagation algorithm [15, 22, 23].

Continuous training allows inclusion of new patterns to the memory permanently. Thus, the training and analysis (diagnosis) are faster and more efficient procedures. Some new elements were added to the training algorithm of the ME-ARTMAP neural network for a correct run of this mechanism.

The first modification is the inclusion of temporary and definitive weights at the $\mathrm{ART}_{\mathrm{a}}$ module. This is necessary to avoid the presence of categories similar to the network memory. Therefore, there are temporary and definitive categories during the process of choosing categories. Further, two choice functions are defined, one for the temporary categories $\left(\boldsymbol{T}_{v}^{t}\right)$, and other for the definitive categories $\left(\boldsymbol{T}_{i}^{d}\right)$ [22].

The temporary category is initialized with only one active neuron, i.e., the first pattern presented to the network $\left(\boldsymbol{W}_{1}^{a t}=\boldsymbol{a}_{1}\right)$, and the weights of the temporary inter-ART module $\boldsymbol{W}^{a b t}$ are set in 1. Definitive weights $\boldsymbol{W}^{a d}, \boldsymbol{W}^{b d}$, and $\boldsymbol{W}^{a b d}$ 


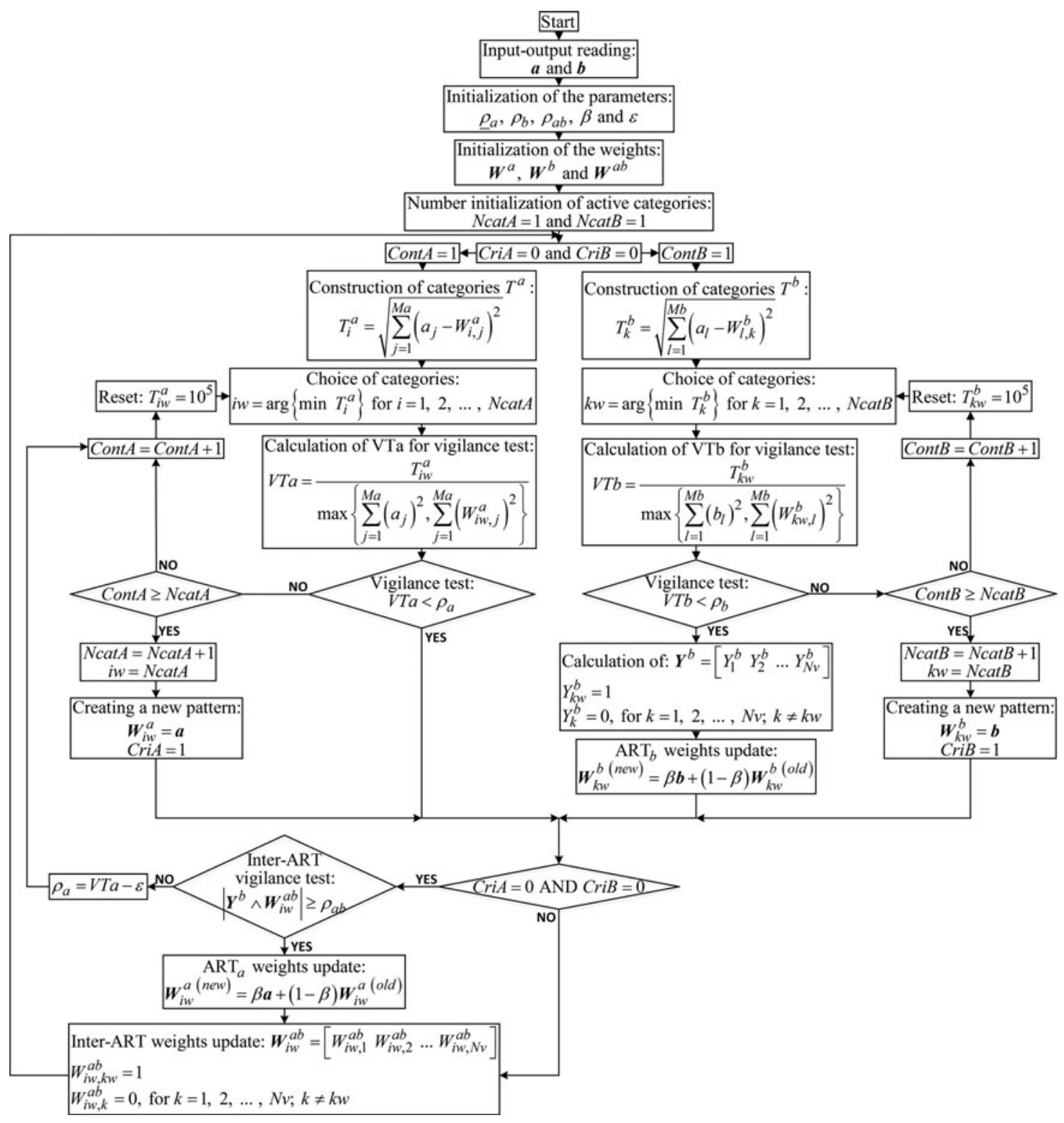

FIGURE 2. Flowchart of the ME-ARTMAP neural network.

of $\mathrm{ART}_{\mathrm{a}}, \mathrm{ART}_{\mathrm{b}}$, and inter-ART are equal to weights $\boldsymbol{W}^{a}, \boldsymbol{W}^{b}$, and $\boldsymbol{W}^{a b}$ of ME-ARTMAP trained off-line, respectively.

The second modification is the inclusion of two new patterns: NMIN and $\eta$. The parameter NMIN (NMIN $\in \mathrm{R}$, with $N M I N>1)$ refers to the quantity of patterns necessary for a temporary weight to become a definitive one. During the training, similar temporary patterns are counted and filed on vector $\boldsymbol{C o n t} \boldsymbol{T}$, and when the value attains NMIN, it becomes definitive. The parameter $\eta(\eta>0)$ verifies if it is necessary to update the winner of the definitive category of weights; i.e., the redundant information are undervalued. Thus, classes and similar patterns do not need to be allocated in different categories, and, moreover, the unnecessary overloading of the memory is avoided [22]. Figure 3 presents the flowchart of the MECT-ARTMAP neural network.

\section{TEST SYSTEM}

Generally, research centers and distribution companies do not have a consistent data set representing the operation system under disturbance. Considering the lack of these registers, it is necessary to use test systems to simulate the disturbances, leading to the extraction of adequate information and validating the disturbance diagnosis methodology. 


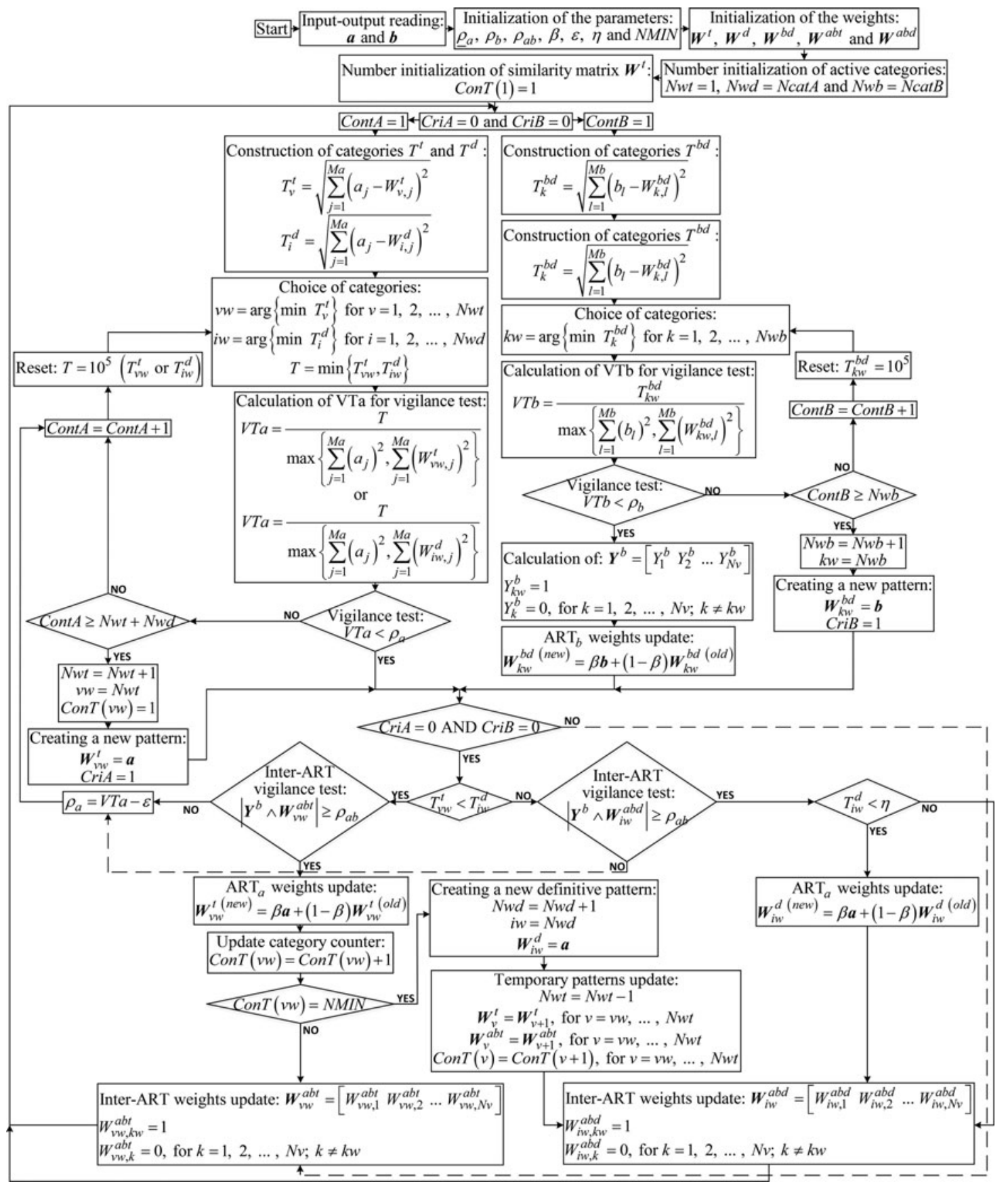

FIGURE 3. Flowchart of the MECT-ARTMAP neural network.

\subsection{Model of the Simulated Feeder}

Therefore, the proposed voltage abnormality diagnosis scheme was tested in a real $13.8-\mathrm{kV}$ distribution system, obtained from [24]. This feeder (test system) was modeled using the software Alternative Transients Program (ATP) [25].

Simulations of the voltage disturbances were executed with one of the routines of the ATP containing the sources of the disturbances approached in this study. Each simulation used a sample frequency of $15.36 \mathrm{kHz}$, corresponding to 256 samples per cycle.

The different signal acquisition devices generally use this sampling frequency. It is emphasized that the neural network accuracy rate will increase along with the number of samples employed in disturbances analysis, i.e., better distinction. 


\begin{tabular}{l|cc}
\multirow{2}{*}{ Parameter } & \multicolumn{2}{|c}{ Decomposition level } \\
\cline { 2 - 3 } & First & Second \\
\hline$\mu$ & 0.005 & 0.07
\end{tabular}

TABLE 2. Parameter for voltage disturbance detection

\section{RESULTS AND DISCUSSIONS}

The methodology developed for voltage disturbance diagnosis in electrical distribution feeders is robust, efficient, and able to learn as time goes by.

The Daubechies mother wavelet [18] is used for detecting the disturbances with a fourth-order filter $(d b 4)$. Table 2 presents the values of the limit parameters used by the operator to verify the operative state of the system. This module presents $100 \%$ accuracy in identifying abnormal situations with high computational performance.

Two cycles under disturbance and two cycles at predisturbance are selected once any abnormal operation on the distribution system is detected and after identifying the instant when the disturbance begins. Subsequently, they are analyzed in four resolution levels by MRA and aggregated by the energy concept. At this step, the mother wavelet is also the Daubechies with the fourth-order filter $(d b 4)$. The input vector of the ME-ARTMAP module (off-line training) uses as reference the normal operation of the energy, referred to as the wavelet coefficients of the pre-disturbance cycles; this energy is subtracted from the energy of the detail and approximation coefficients of the cycles under disturbance.

At the classification step, there are 3024 voltage disturbance vectors of which 524 were selected for training the ME-ARTMAP module and 2500 for test. It is emphasized that this selection was done pseudo-randomly (with a predefined seed). Additionally, 432 disturbances from the harmonic class were included on the test pattern set. Thus, it was possible to visualize the capacity of the continuous training module in acquiring new knowledge, showing the improvement of the

\begin{tabular}{l|cc} 
Voltage disturbance & Pattern test & Accuracy (\%) \\
\hline Swell & 346 & 83.51 \\
Sag & 338 & 54.73 \\
Interruption & 338 & 81.95 \\
Harmonics & 432 & 0 \\
Swell with harmonics & 359 & 93.31 \\
Sag with harmonics & 346 & 54.33 \\
Oscillatory transient & 341 & 99.70
\end{tabular}

TABLE 3. Accuracy rate without the continuous training module

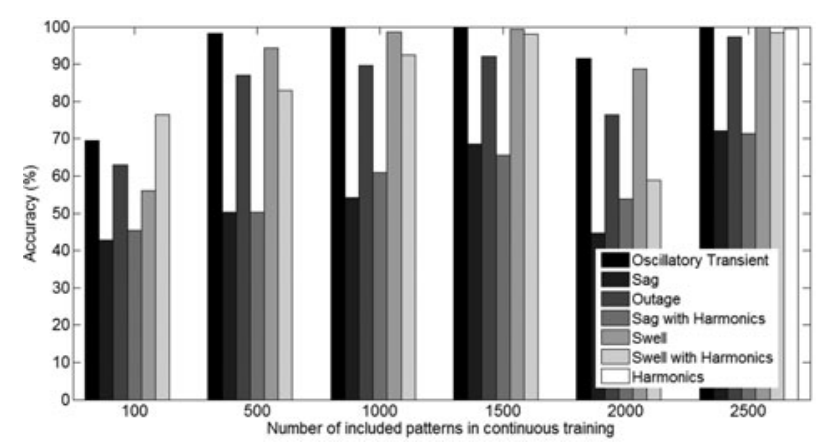

FIGURE 4. Evolution of the neural network with continuous training.

results as time passed. Table 3 shows the accuracy rate in the ME-ARTMAP module.

It is observed that by analyzing the accuracy percentage for each one of the disturbances, this percentage is zero for the voltage disturbance defined as harmonic. This occurrence is because of the lack of patterns representing the harmonic disturbance during the training of the neural network; i.e., there is no harmonic disturbance on the off-line training of the neural network. Thus, the neural network is unable to learn such a disturbance, and when they are presented at the test step, the neural network classifies wrongly; therefore, the accuracy rate for this disturbance is zero.

Furthermore, the accuracy rate of the disturbances sag and sag with harmonic was very low. The errors in classifying these disturbances are because of the characteristic index, once the disturbances sag, outage, and sag with harmonic present the same behavior in amplitude. Therefore, the disturbances sag and sag with harmonic are classified as outage by the classification module.

Finally, at the off-line training step, the continuous training module is activated. Each one of the test pattern used at the ME-ARTMAP is presented separately, i.e., one by one, for the MECT-ARTMAP. The MECT-ARTMAP provides a new diagnosis even as new patterns are presented to the network. Then, considering every test pattern, the network provides 2500 different diagnoses. Figure 4 presents the evolution of the accuracy rate after including each one of the test patterns on the continuous training. Table 4 shows the final result after including the 2500 patterns on the continuous training.

Comparing Tables 3 and 4, it is observed that there is an improvement in the classification step; i.e., the neural network with continuous training is able to classify correctly disturbances that were wrongly classified previously. It is also observed that the accuracy of the disturbance harmonic is improved from 0 to 99.53 . The increase of this accuracy rate is a consequence of the neural network incorporating new patterns 


\begin{tabular}{l|cc} 
Voltage disturbance & Pattern test & Accuracy (\%) \\
\hline Swell & 346 & 100 \\
Sag & 338 & 72.18 \\
Interruption & 338 & 97.33 \\
Harmonics & 432 & 99.53 \\
Swell with harmonics & 359 & 98.32 \\
Sag with harmonics & 346 & 71.38 \\
Oscillatory transient & 341 & 99.70 \\
Total & 250 & 91.20
\end{tabular}

TABLE 4. Accuracy rate with the continuous training module

into the data base, i.e., disturbances that were previously unknown are now correctly identified at the test step. This case exemplifies what occurs if an unknown disturbance were presented to the ARTMAP-EMTC; i.e., first, it cannot identify correctly, but after verifying the need to learn this unknown data, the neural network includes this pattern automatically to the memory and is able to correctly identify a posteriori.

The MECT-ARTMAP presented efficiency and robustness to the dynamism of the electrical power systems; i.e., disturbances were detected and classified at different loading levels and locations (bus) of the distribution system. This improvement process is observed even as the neural network executes the diagnosis simultaneously with the continuous training. The parameters used at the training and test phases are training rate $\beta=1$; vigilance parameters $\rho_{a}=0.15, \rho_{b}=0$, and $\rho_{a b}=$ 0.95 ; incremental vigilance parameter $\varepsilon=0.01$; novelty index $\eta=2$; and similarity pattern $N M I N=2$.

\section{CONCLUSION}

This study consists of a neural structure with continuous training applied to classify voltage disturbances in electrical distribution systems. This decision-making tool aids the substation automation process to restore normal operation in a faster, flexible, and robust manner. The voltage disturbance diagnosis module is projected to attend the needs of the distribution companies, considering the current economic situation of the electrical sector, and can be easily implemented with reduced costs, as it is only necessary to acquire equipment for signal acquisition and a data processing module.

By the combined use of signal processing techniques and methodologies based on artificial intelligence, the diagnosis system presents high generalization capacity, flexibility, and efficiency-important characteristics in aiding the decision making at substations. The detection process is able to identify every abnormal operational situation. Thus, once the presence of a voltage disturbance is identified, the voltage oscil- lographies are analyzed by the MRA and the energy concept, generating characteristic indices that represent the detected disturbances. Therefore, these indices are presented to the MECT-ARTMAP neural network providing the operator the type of voltage disturbance. Furthermore, using a new different training concept, the neural module at each diagnosis improves or acquires new knowledge.

Therefore, the advantage of the MECT-ARTMAP neural network in relation to other conventional neural networks available on the literature is that it allows continuous improvement of the existent categories with the learning being continuously realized without the need to reinitialize the training. This important characteristic allows the application of the neural network in modern electrical energy systems, i.e., smart grids, once it has the capacity to learn in real time.

\section{FUNDING}

The authors are grateful to the Coordination for the Improvement of Higher Level Personnel (CAPES) and São Paulo Research Foundation (FAPESP) for supporting this research.

\section{REFERENCES}

[1] Fan, J., and Borlase, S., "The evolution of distribution," IEEE Power Energy Mag., Vol. 7, No. 2, pp. 63-68, March-April 2009.

[2] McDonald, J. D., Electric Power Substations Engineering, 2nd ed., Boca Raton: CRC Press, 2003.

[3] Northcote-Green, J., and Wilson, R., Control and Automation of Electrical Power Distribution Systems, 1st ed., Boca Raton: Taylor \& Francis, 2003.

[4] Kezunovic, M., "Intelligent applications in substations: disturbance analysis," Proceedings of the IEEE Power Engineering Society General Meeting, pp. 719-723, New York, 6-10 June 2004.

[5] Panigrahi, B. K., and Pandi, V. R., "Optimal feature selection for classification of power quality disturbances using wavelet packet-based fuzzy $k$-nearest neighbor algorithm," IET Generat. Transm. Distribut., Vol. 3, No. 3, pp. 296-306, 2012.

[6] Venkatesh, C., Siva-Sarma, D. V. S. S., and Sydulu, M., "Classification of voltage sag, swell and harmonics using S-transform based modular neural network," 14th International Conference on Harmonics and Quality of Power (ICHQP), pp. 1-7, Bergamo, Italy, 26-29 September 2010.

[7] Granados-Lieberman, D., Romero-Troncoso, R. J., OsornioRios, R. A., Garcia-Perez, A., and Cabal-Yepez, E., "Techniques and methodologies for power quality analysis and disturbances classification in power systems: A review," IET Generat. Transm. Distribut., Vol. 5, No. 4, pp. 519-529, 2011.

[8] Gaouda, A. M., Salama, M. M. A., Sultan, M. R., and Chikhani, A. Y., "Power quality detection and classification using waveletmultiresolution signal decomposition," IEEE Trans. Power Del., Vol. 14, No. 4, pp. 1469-1476, 1999. 
[9] Abdelsalam, A. A., Eldesouky, A. A., and Sallam, A. A., "Characterization of power quality disturbances using hybrid technique of linear Kalman filter and fuzzy-expert system," Electr. Power Syst. Res., Vol. 83, No. 1, pp. 41-50, 2012.

[10] Dehghani, H., Vahidi, B., Naghizadeh, R. A., and Hosseinian, S. H., "Power quality disturbance classification using a statistical and wavelet-based hidden Markov model with Dempster-Shafer algorithm," Electr. Power Syst. Res., Vol. 47, pp. 368-377, 2013.

[11] Huang, N., Xu, D., Liu, X., and Lin, L., "Power quality disturbances classification based on S-transform and probabilistic neural network," J. Neurocomput., Vol. 98, pp. 12-23, 2012.

[12] Rodríguez, A., Aguado, J. A., Martín, F., López, J. J., Muñoz, F., and Ruiz J. E., "Rule-based classification of power quality disturbances using S-transform," Electr. Power Syst. Res., Vol. 86, pp. 113-121, 2012.

[13] Eristi, H., and Demir, Y., "Automatic classification of power quality events and disturbances using wavelet transform and support vector machines," IET Generat. Transm. Distribut., Vol. 6, No. 10, pp. 968-976, 2012.

[14] Nguyen, T., and Liao, Y., "Power quality disturbance classification utilizing S-transform and binary feature matrix method," Electr. Power Syst. Res., Vol. 79, pp. 569-575, 2009.

[15] Marchiori, S. C., Silveira, M. C. G., Lotufo, A. D. P., Minussi, C. R., and Lopes, M. L. M., "Neural network based on adaptive resonance theory with continuous training for multi-configuration transient stability analysis of electric power systems," Appl. Soft Comput., Vol. 11, No. 1, pp. 706-715, 2011.

[16] Decanini, J. G. M. S., Tonelli-Neto, M. S., Malange, F. C. V., and Minussi, C. R., "Detection and classification of voltage disturbances using a fuzzy-ARTMAP-wavelet network," Electr. Power Syst. Res., Vol. 81, No. 12, pp. 2057-2065, 2011.

[17] Mallat, S., A Wavelet Tour of Signal Processing: The Sparse Way, 3rd ed., Burlington: Academic Press, 2009.

[18] Daubechies, I., Ten Lectures on Wavelets, 1st ed., Philadelphia: Society for Industrial and Applied Mathematics, 1992.

[19] Oppenheim, A. V., and Schafer, R. W., Digital Signal Processing, Englewood Cliffs: Prentice-Hall, 1975.

[20] Decanini, J. G. M. S., Tonelli-Neto, M. S., and Minussi, C. R., "Robust fault diagnosis in power distribution systems based on fuzzy ARTMAP neural network-aided evidence theory," IET Generat. Transm. Distribut., Vol. 6, No. 11, pp. 1112-1120, 2012.

[21] Carpenter, G. A., Grossberg, S., Markuzon, N., Reynolds, J. H., and Rosen, D. B., "Fuzzy ARTMAP: A neural network architecture for incremental supervised learning of analog multidimensional maps," IEEE Trans. Neural Networks, Vol. 3, No. 5, pp. 698-713, 1992.

[22] Moreno, L. A., Transient Stability Analysis by a Modified Euclidean ART-ARTMAP Fuzzy Neural Network with Continuous Training, PhD. Thesis, UNESP, Univ Estadual Paulista, Ilha Solteira, SP, Brazil, 2010 (in Portuguese).

[23] Vuskovic, M., and Du, S., "Classification of prehensile EMG patterns with simplified fuzzy ARTMAP," IEEE Proc. Int. Joint Conf. Neural Networks, Vol. 3, No. 10, pp. 2539-2544, 2002.
[24] Pereira, R. A. F., Silva, L. G. W., Kezunovic, M., and Mantovani, J. R. S., "Improved fault location on distribution feeders based on matching during-fault voltage sags," IEEE Trans. Power Del., Vol. 24, No. 2, pp. 852-862, 2009.

[25] SINTEF Energy Research, Alternative Transients Program (ATP), RuleBook, Trondheim, Norway: SINTEF Energy Research, 2007.

\section{BIOGRAPHIES}

Ana Claudia Barros received her B.Sc. in mathematical science from Universidade Federal de Mato Grosso do Sul, Três lagoas, Mato Grosso do Sul (MS), Brazil, in 2005. She received her M.Sc. and Ph.D. from UNESP, Univ. Estadual Paulista, São Paulo, Brazil, in 2009 and 2013, respectively. Currently she works at the Anhanguera University (Uniderp), Campo Grande, MS, Brazil, as an assistant professor. Her fields of interest are electrical distribution system fault and mathematic modeling applications to electrical power systems.

Mauro S. Tonelli-Neto received his B.Sc., M.Sc., and Ph.D. in electrical engineering from UNESP, Universidade Estadual Paulista, Ilha Solteira (SP), Brazil, in 2009, 2012, and 2015 , respectively. He is an assistant professor in Instituto Federal de Educação, Ciência e Tecnologia de São Paulo, Votuporanga (SP), Brazil. His fields of interest are electric power distribution system operation, fault diagnosis, and intelligent systems (artificial neural networks and fuzzy $\operatorname{logic})$.

José Guilherme Magalini Santos Decanini received his B.S., M.S., and Ph.D. from the Electrical Engineering Department, UNESP, Univ Estadual Paulista, Ilha Solteira, São Paulo, Brazil, in 2005, 2008, and 2012, respectively. Currently he works at the Instituto Federal de Educação, Ciência e Tecnologia de São Paulo, Votuporanga, São Paulo, Brazil, as an assistant professor. His research interests are operation of distribution systems and intelligent systems.

Carlos Roberto Minussi received his electrical engineering degree from Universidade Federal de Santa Maria, Santa Maria, Brazil, in 1978, and his M.Sc. and Ph.D. from Universidade Federal de Santa Catarina, Brazil, in 1981, and 1990, respectively. His research interests are transient stability analysis, load forecasting, dynamic and preventive control, electrical power systems faults, artificial neural networks, fuzzy logic, and artificial immune system applications. 